\section{CPC-083 MEDICATION RECONCILIATION EXPERIENCE IN PSYCHIATRIC HOSPITALS, SAUDI ARABIA}

doi:10.1136/ejhpharm-2013-000276.540

K Aljumah. AL Amal Psychiatric Hospital, Department of Pharmacy, Riyadh, Saudi Arabia

Background In 2006, the Joint Commission on Accreditation of Healthcare Organizations (JCAHO) started the new year with a mandate for accredited organisations to implement an innovative initiative: Medicines Reconciliation. The mandate attempted to address the 1.3 million iatrogenic adverse events that occur annually, many of which are related to medicines.

Medicines reconciliation is an effective process of reducing errors and harm associated with loss of medicines information, as patients transfer between wards (handovers). It may prevent up to $70 \%$ of all potential errors and $15 \%$ of all adverse drug events.

Literature investigation of medicines reconciliation is minimal in psychiatric hospitals.

Limited information is available about medicines reconciliation in psychiatric hospitals in Saudi Arabia.

Purpose To gain an insight into pharmacists' practise, knowledge and attitudes toward medicines reconciliation in psychiatric hospitals and the most common challenges and barriers.

Materials and Methods We developed and administered a survey to the Director of Pharmacy at all psychiatric hospitals in Saudi Arabia (20 hospitals), The questionnaire was modified after piloting on 10 randomly-selected pharmacists working in psychiatric hospitals. The survey included scales measuring (1) pharmacists' attitudes towards medicines reconciliation, (2) pharmacists awareness of medicines reconciliation and (3) local practise in Saudi psychiatric hospitals.

Results Response rate: $90 \%$ of pharmacy directors in psychiatric hospitals in Saudi Arabia returned the survey, 70\% indicated that they were familiar with the concept of medicines reconciliation and believed that medicines reconciliation represented an important safety intervention. Only $25 \%$ of pharmacy director had initiated medicines reconciliation in practise, and $40 \%$ did not believe that they had the necessary resources to manage discrepancies.

Conclusions Pharmacists had mixed attitudes toward implementation of medicines reconciliation services due to the limited patient transfer between wards or between care (acute and ambulatory) in psychiatric hospitals; on the other hand they believed that medicines reconciliation would improve patient safety and result in a better therapeutic outcome. Pharmacists were willing to practise medicines reconciliation if they could be trained.

No conflict of interest.

\section{CPC-084 MEDICATION REVIEWS BY CLINICAL PHARMACISTS AT HOSPITALS LEAD TO IMPROVED PATIENT OUTCOMES: A SYSTEMATIC REVIEW}

doi:10.1136/ejhpharm-2013-000276.541

'T Graabæk, '2LJ Kjeldsen. 'Hospital South West Jutland, Emergency Department, Esbjerg, Denmark; ${ }^{2}$ Amgros I/S, The Research Unit for Hospital Pharmacy, Copenhagen, Denmark

Background Suboptimal use of medicines may lead to morbidity, mortality and increased costs. In order to reduce unnecessary patient harm, an increasing number of hospitals have implemented pharmaceutical care interventions such as medicines reviews. Some recent studies indicate a positive effect of pharmacist-led medicines reviews in hospitals, but the quality and outcome measures vary among studies. Hence there is a need to compile evidence within this area.

Purpose To identify, assess and summarise the literature investigating the effect of pharmacist-led medicines reviews in hospitalised patients.
Materials and Methods Five databases were searched from their inception to 2011: MEDLINE, EMBASE, CINAHL, Web of Science (including a citation search of relevant papers) and the Cochrane Library. Relevant systematic reviews and personal archives were also hand-searched for studies for inclusion. Only original research papers published in English describing pharmacist-led medicines reviews in a hospital setting including a minimum of 100 patients were included in the final assessment.

Results A total of 836 research papers were identified and 30 publications were included in the study. Twenty studies were descriptive studies while ten studies were controlled to some extent. Only six studies were randomised controlled trials. Generally, the interventions were well implemented with acceptance rates between $39-100 \%$. The key findings indicated positive effects on quality of prescribing, quality of life, readmission rates and emergency department visits, time to readmission and costs. However, no effect on survival rates was found in addition to several other statistically insignificant results.

Conclusions Only a few papers describing pharmacist-led medicines reviews in the hospital setting were designed as randomised controlled trials and were evaluated using hard endpoints. Future research within this area should be designed using rigorous methodology and include outcome measures for patient health outcomes.

No conflict of interest.

\section{CPC-085 MEDICINES AND THEIR COSTS IN THE LAST SIX DAYS OF LIFE}

doi:10.1136/ejhpharm-2013-000276.542

P Tavcar, M Sonc, M Fortuna Luzar, A Eberl, I Virant, S Rozman. Institute of Oncology Ljubljana Slovenia, Pharmacy, Ljubljana, Slovenia

Background Palliative care provides many advantages to patients who face life-threatening illness. The five most common symptoms that usually occur in the last days of life are pain, nausea and vomiting, restlessness, dyspnoea and respiratory tract secretions. Supportive treatment for pain and symptom relief should be incorporated into treatment to address these issues.

Purpose The aim of this retrospective study was to compare the use of medicines in the last six days of life in patients treated according to a palliative or standard treatment pathway.

Materials and Methods Inclusion criteria were treatment at the Institute of Oncology, Ljubljana, Slovenia, within the last 6 days of life and the diagnosis of advanced or metastatic cancer. 25 patients were included in the palliative treatment pathway, whereas 25 were treated according to standard treatment pathway and served as a control group. Both groups were comparable in terms of the primary tumour site and median age of the patients.

Results The majority of patients in both groups received strong opioid analgesics. Other medicines to relieve symptoms, such as haloperidol, midazolam, dexamethasone, butylscopolamine and metoclopramide, were more likely to be administered in the palliative group. Polypharmacy was a common problem observed in both groups. However, patients treated according to the palliative treatment pathway received on average 10 medicines, whilst those in control group 14. The cost of medicines was 2.7-fold lower in the palliative group, $15 €$ compared to $42 €$ per patient per day. The difference was mainly attributed to unnecessary prescribing of low-molecular weight heparins, systemic antimicrobial medicines and parenteral nutrition.

Conclusions Palliative care is given to improve the quality of life of patients with serious or life-threatening disease, such as cancer. The goal is to prevent the symptoms and side effects of advanced disease, and not to cure. In our opinion, the essential medicines in palliative setting are analgesics, antiemetics, sedatives, anxiolytics and anticholinergics. Other, unnecessary, medicines should be omitted from the treatment.

No conflict of interest. 